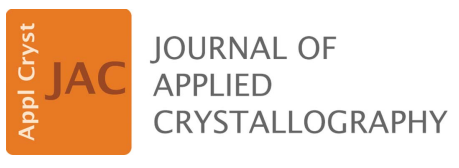

ISSN 1600-5767

Received 26 February 2018

Accepted 4 July 2018

Edited by J. M. García-Ruiz, Instituto Andaluz de Ciencias de la Tierra, Granada, Spain

₹ These authors contributed equally to the work.

Keywords: science laboratories; research experience; crystal growth; $\mathrm{X}$-ray crystallography; education.

Supporting information: this article has supporting information at journals.iucr.org/j

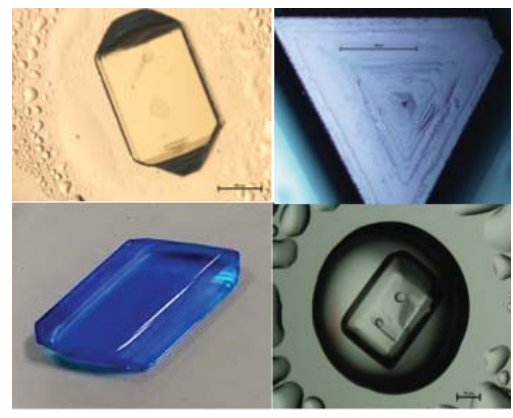

C 2018 International Union of Crystallography

\section{Crystal growth, a research-driven laboratory course}

\author{
Jamie Whelan, ${ }^{a} \ddagger$ Joseph Koussa, ${ }^{\mathrm{a}}$ Ibrahim Chehade, ${ }^{\mathrm{a}}$ Merima Sabanovic, ${ }^{\mathrm{a}}$ Adrienne \\ Chang, ${ }^{a}$ Daniel Carelli, ${ }^{a}$ Zhihua An, ${ }^{b, c}$ Lu Zhang, Joel Bernstein ${ }^{\mathrm{a}, \mathrm{b}, \mathrm{d}}$ and Wael M. \\ Rabeh $^{\mathrm{a} *} \neq$
}

\begin{abstract}
${ }^{\mathbf{a}}$ Faculty of Science, New York University Abu Dhabi, Abu Dhabi, United Arab Emirates, ${ }^{\mathbf{b}}$ Faculty of Science, New York University Shanghai, Pudong, Shanghai, People's Republic of China, 'Department of Chemistry, New York University, New York, NY 10003, USA, and 'Department of Chemistry, Ben-Gurion University of the Negev, Beer Sheva, 84120, Israel. *Correspondence e-mail: wael.rabeh@nyu.edu
\end{abstract}

The research-driven laboratory experiment described herein has at its core the individual development of students, combining core subject matter with the opportunity to explore, in a research environment, areas outside of traditional curricula; however, it maintains the pedagogical training for an undergraduate major degree in chemistry and sciences in general. The laboratory can feasibly be implemented in high schools to expose students to an engaging and intellectually fulfilling aspect of chemistry early in their career. This seven-week project is based on the growth and study of crystals and encourages students, from the outset, to conceive, propose, design, plan and carry out their own research on chemicals and conditions of their own choosing. The wide array of laboratory equipment, analytical instrumentation and techniques that the students are potentially exposed to, from micropipettes and optical microscopes to scanning electron microscopy and powder X-ray diffraction, puts these projects on a par with senior capstone research projects. The feedback from the students for the seven years this project has been in operation is almost unanimous in enthusiasm and praise.

\section{Introduction}

As defined by the International Union of Crystallography (IUCr), a material is a crystal if it has essentially a sharp diffraction pattern. The smallest repeating collection of components is termed the 'unit cell', the crystal building block, and the way in which these blocks are organized together determines the shape and size of a crystal. The purpose of the crystal growth project presented here is to enhance the overall learning experience of chemistry laboratory courses and to engage undergraduate science and engineering students in the design and execution of their own research projects (Grazulis et al., 2015). It is also expected that with slight modifications this project can be easily incorporated into high schools as a stand-alone chemistry experiment or a more integrative project spanning several scientific disciplines. Here, we show how the design of a seven-week course around crystals and crystal growth enables students to incorporate their scientific preferences into their own individual project and even experience an introduction to research science.

Traditional general chemistry and science laboratory experiments tend to follow the same approach, where students are introduced to the topic to be studied through some visual aids (board, PowerPoint etc.). Next, the procedure is explained in sufficient detail that the students can carry out the experiment with little or no intellectual input; following this, students 
Table 1

List of chemical compounds for the setup of macro- and microcrystals.

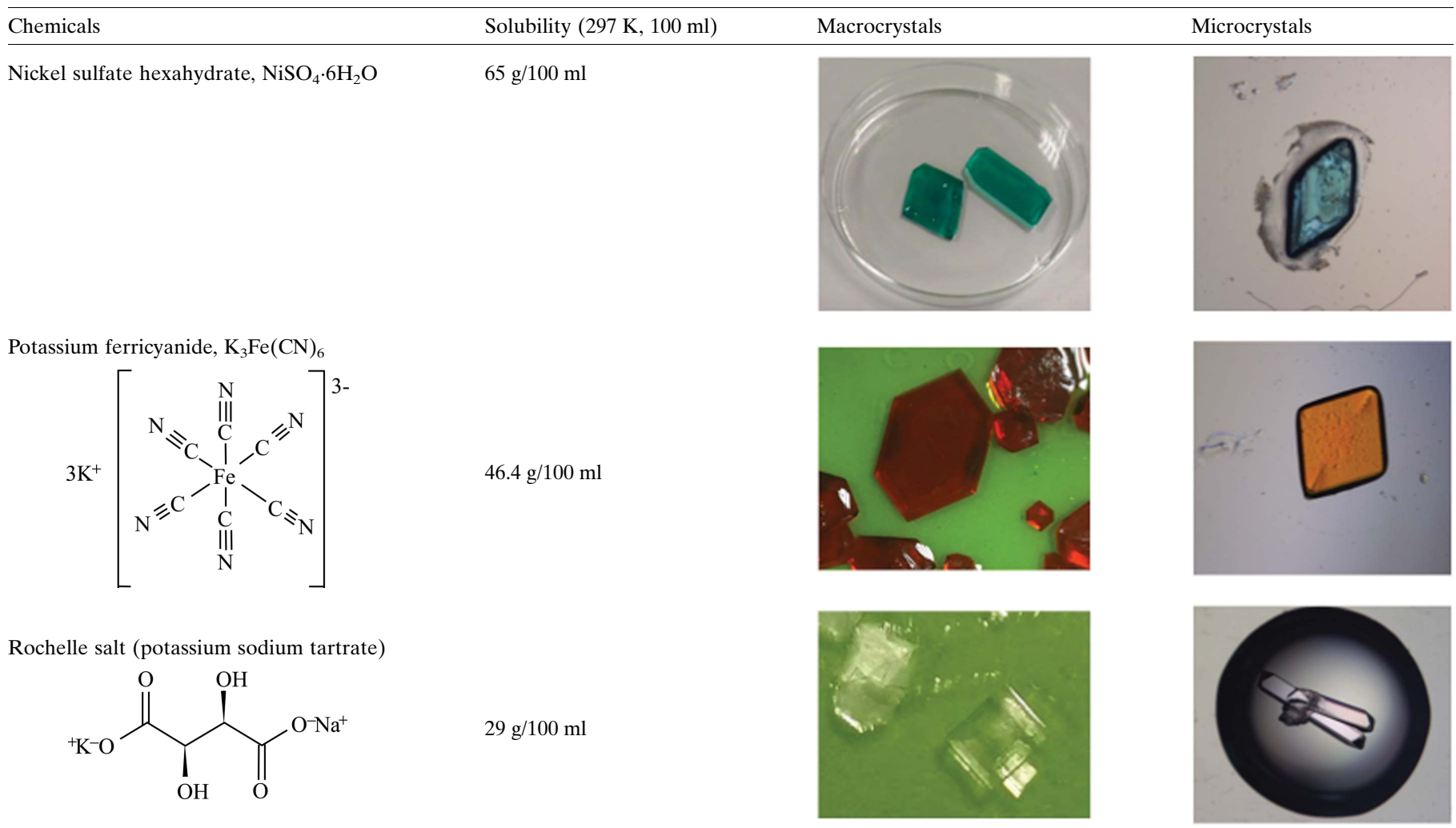

are given an experimental procedure (e.g. handout) to follow which details the step-by-step process to reach the end goal of the experiment. Lastly, some discussion ensues regarding the results to be obtained and how to process them. Throughout the experiment, an instructor is present in the laboratory, answering questions, fixing experimental setups and correcting mistakes, until the end of the allotted time, at which point the students clean up, leave and at some later stage write up a laboratory report which follows a pre-set template.

This format exists all over the world, in many schools and in many undergraduate university courses, the presumed purpose being to train students how to work in a laboratory, design experiments, problem solve and develop critical thinking to name but a few. In fact, the above description of a laboratory experiment does not deviate much from a culinary novice following a recipe from a YouTube channel, with all the cognitive development to match (Domin, 1999a,b). The result in schools is that chemistry laboratories can be perceived as being 'dull' (Hodson, 1990), an attitude universities and high schools must strive to combat.

Over the years, much work has focused on trying to remedy the chemistry laboratory outcomes to better prepare students for chemistry specifically and research in general. The perceived lack of desired learning achieved from chemistry laboratory courses has led to one study challenging places of learning to re-evaluate the role of the laboratory course in order justify their position in the programs (Reid \& Shah, 2007).
Inquiry-based learning was the model and driving force to build the crystal growth laboratory described herein, as demonstrated in other successful laboratory exercises (Grazulis et al., 2015). The choice of an appropriate project was inspired by the 1960 book Crystals and Crystal Growing by Holden and Singer (HS) and was intended to excite students about science (Holden \& Singer, 1960; Holden \& Morrison, 1982; Dhanaraj et al., 2010; Douglas \& Ho, 2007; Byrappa \& Ohachi, 2003; Sunagawa \& Suchtelen, 1995; Sunagawa, 2005). A similar experience has been introduced by the IUCr through a crystal growing competition for schoolchildren, which highlights the importance of incorporating inquiry-based learning into school curriculums (IUCr, 2018). Expanding on the HS approach, the goals for our crystal project revolve around crystal growth, with many of the specific details left up to the students themselves. Thus, for example, one group could look at modifying crystal formation while another could maximize crystal growth.

The laboratory experiment explained herein has at its core the individual development of students, combining subject matter with the opportunity to explore, in a research-like environment, areas outside of the traditional curriculum, although the teaching and practice of many laboratory techniques are incorporated. Thus, it maintains the pedagogical training for an undergraduate major degree in chemistry and can feasibly be implemented in high schools to expose students to an engaging and intellectually fulfilling aspect of chemistry early in their career (Supplementary Note 1). 
If the students do not have the required laboratory skills or safety instruction, multiple short standard laboratory exercises can be implemented at the beginning to build the students' safety habits and laboratory skills. Successively, the sevenweek crystal growth project aims to encourage students from the outset to conceive, propose, design, plan and carry out their own research on chemicals and conditions of their own choice. The wide array of laboratory equipment and analytical instrumentation and techniques that the students are potentially exposed to, from micropipettes and optical microscopes to scanning electron microscopy and powder X-ray diffraction, puts these projects on a par with a senior capstone research project. The laboratory project can also be tailored for institutions with limited equipment resources. The feedback from the students for the seven years this project has been in operation is almost unanimous in enthusiasm and praise, especially when compared to traditional chemistry laboratory experiments.

At the conclusion of the crystal laboratory, each group (usually pair) of students prepares a poster to summarize their work (samples of students' posters are included in the supporting information); a crystal growth symposium crowns the course, where each group gives an introductory 'thumb sketch' presentation to introduce their project prior to the formal poster session. To accommodate a large size class, short video clips prepared by the students are shown prior to the formal poster session [e.g. the 5th Crystal Growth Symposium held at New York University Abu Dhabi (NYUAD) in the spring of 2015; https://www.youtube.com/watch?v=4e9BC18Dyfk]. Faculty members and instructors from the departments of physics, chemistry, biology and engineering are invited to serve as judges for the best poster competition, which has turned out to be quite competitive, considerably raising the level of achievement and student enthusiasm and satisfaction across the board. Scores from the judges are incorporated into the students' final grades.

\section{Laboratory experiment}

The crystal growth project is composed of two parts: the growth of inorganic macrocrystals, and the growth of small inorganic or protein microcrystals. The differences between the two techniques are the crystal growth setup and the amount of chemical used in each experiment. The equipment required for the crystal project is the same as for any basic chemistry laboratory course unless specific equipment is required for a particular student's project. Hot plates and stirrers are common for all, as are beakers, conical flasks, spatulas, mass balances etc. Micropipettes and pipette tips, as well as 24-well crystallization plates, are primarily used for microcrystal projects. Stereomicroscopes are used for visual analysis of crystals (generally microcrystals). The crystal growth laboratory can be split into two different parts (macro- and microcrystal growth) that can also be adapted as two separate projects depending on the resources available to the students. Table 1 lists different examples of chemicals that crystallize as macro- and microcrystals.

Students grow large-size macrocrystals from saturated or supersaturated chemical solutions (Table 1 and Fig. 1). A detailed protocol for macrocrystal growth is included in Supplementary Note 2, along with a list of recommended chemicals, their water solubilities and the approximate cost (Table S1). The macrocrystal laboratory requires less equipment and fewer consumables than the microcrystal laboratory; although larger quantities of chemicals are needed to grow the large macrocrystals, these can be relatively inexpensive.

Protein crystals, used in structural biology to study the molecular structure and fold of proteins, require different crystal growth conditions. A protein solution cannot reach saturation as proteins will aggregate and precipitate out of solution. As a result, a vapor diffusion method is employed in the growth of protein crystals, using multiple crystallization solutions. Here, the hanging-drop vapor diffusion method was used for the crystallization of inorganic or protein crystals (McPherson, 2004; Supplementary Note 2). Depending on the plate well size used (typically $22 \mathrm{~mm}$ diameter), up to three different chemicals can be screened in one trial to maximize the number of samples screened in one plate, and a 

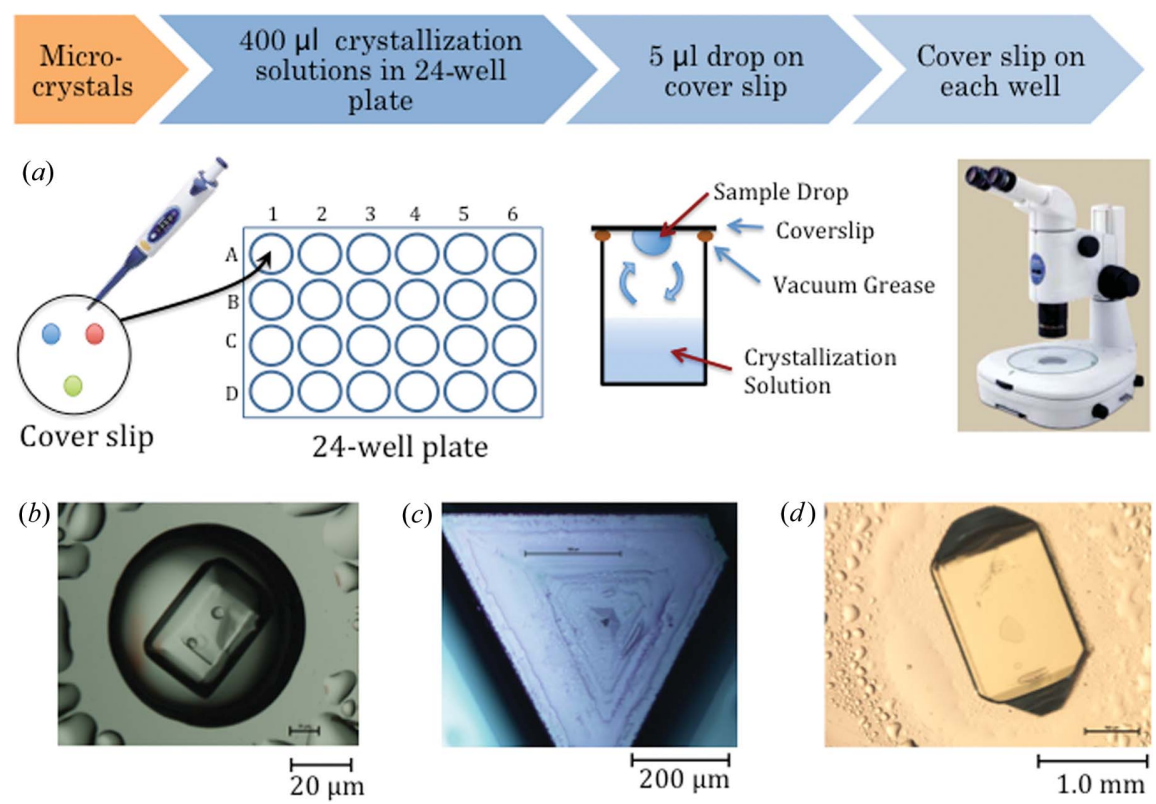

Figure 2

Microcrystal growth. (a) The same chemical compounds used in macrocrystal growth can also be screened for microcrystal growth using the hanging-drop vapor diffusion method. Microcrystals of $(b)$ aluminium potassium sulfate dodecahydrate, $(c)$ chromium potassium sulfate dodecahydrate and $(d)$ potassium dihydrogen phosphate. The size scales are indicated below each image.

stereomicroscope is used to optically survey the crystals produced under certain crystallization conditions, ranging in size from $50 \mu \mathrm{m}$ up to $5 \mathrm{~mm}$ (Table 1 and Fig. 2).

\subsection{Representative student projects}

We present here two typical student projects. In the first, Merima Sabanovic and Adrienne Chang, freshmen students at NYUAD majoring in biology and chemistry, respectively, carried out a project entitled Alkalization of Urea, Oxalate, and Newberyite Crystals Grown In Vitro as a Potential Treatment and Prevention of Bladder Stones. Urolithiasis, the formation of urinary stones, is one of the leading health issues in cats and dogs, and results from the supersaturation of urine with minerals such as calcium or magnesium (Syme, 2012; Sun et al., 2010). Calcium oxalate is the most common urinary stone, followed by calcium phosphate, triple phosphates and uric acid (Smith \& St Peter, 2007; Khan, 1997). Diagnosis of bladder stones is done through palpatory examination, ultrasound or X-ray imaging after the application of an iodinebased contrast medium that is required for the detection of radiolucent crystals (Rao et al., 2011). Bladder stones or calculi can be removed surgically, and smaller stones are treated through diet and antibiotics (Syme, 2012). Other treatments, such as bicarbonate tablets, are directed to alkalize the urine and dissolve and/or prevent the growth of crystals potentiated at low $\mathrm{pH}$.

While researchers use biologically extracted bladder stones for experimental endeavors, this project investigated the in vitro growth of urinary stones, a topic with limited prior findings. Struvite stones (ammonium magnesium phosphate;

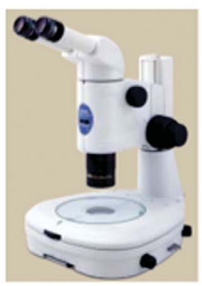

$\mathrm{NH}_{4} \mathrm{MgPO}_{4}$ ) are urinary calculi that are caused by urease-producing gram-negative bacteria, where the enzyme, urease, metabolizes urea into ammonia and carbon dioxide (Miano et al., 2007). Ammonium ions bind phosphate and magnesium to form struvite, which is highly insoluble. The presence of high levels of carbon dioxide alkalinizes the urine and enhances the formation of struvite stones (Miano et al., 2007). Although the growth of struvite microcrystals by a simple diffusion technique in agar gel was not successful, one of its analogs, struvite- $\mathrm{K}$ (potassium magnesium phosphate hexahydrate; $\mathrm{KMgPO}_{4} \cdot 6 \mathrm{H}_{2} \mathrm{O}$ ), gave crystals and was used for further analysis (Figs. $3 a$ and $3 b$ ) (Sun et al., 2010; Suryawanshi \& Chaudhari, 2014; Henisch, 1996). The effect of variation of $\mathrm{pH}$ on the growth of struvite-K crystals was investigated to test the potential preventive effects of alkalization on stone formation. In-gel crystallization trials were set up by dissolving the first active ionic solution (used to form the crystal of interest) in a hot agar solution, which was then left to solidify overnight in a half-filled glass test tube (Fig. 3a) (Henisch, 1996). The second chemical solution was placed on top of the agar, covered with

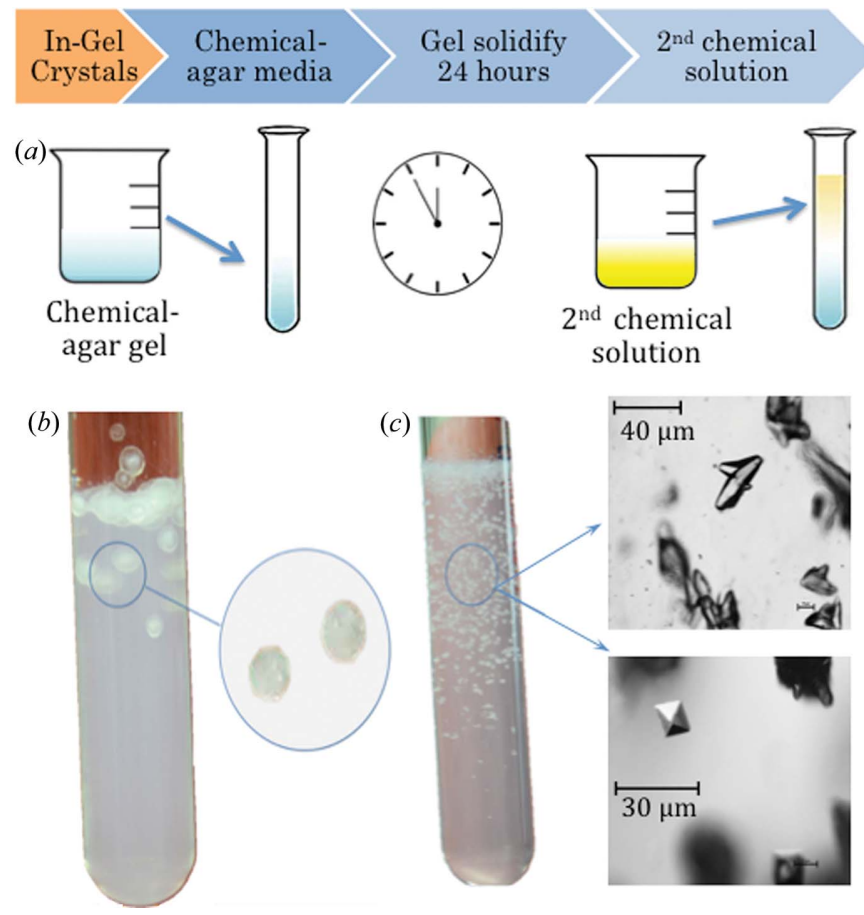

Figure 3

In-gel crystal growth. (a) The single diffusion technique is used to grow a crystal in gel, where one chemical is dissolved in hot agar and the other in water to be placed on top of the chemical-agar gel. In-gel crystals of $(b)$ struvite-K and (c) strontium oxalate observed using a stereomicroscope. 
Parafilm to limit its evaporation, and set vertically to initiate crystal formation. For struvite-K crystals, an in-gel solution of potassium dihydrogen phosphate $\left(\mathrm{KH}_{2} \mathrm{PO}_{4}\right)$ was covered with a solution of magnesium acetate, both at $1.5 \mathrm{M}$ (Fig. $3 b$ ) (Suryawanshi \& Chaudhari, 2014). Crystals of struvite-K did not grow at $\mathrm{pH} 7$ or 10 , possibly because of the large volume of $\mathrm{NaOH}$ needed for alkalization of the ionic solution; thus, the solution was too dilute for crystallization during the project's time frame. The 'stones' formed under acidic condition by addition of $0.25 \mathrm{M} \mathrm{HCl}$ were confirmed by powder X-ray diffraction (PXRD) analysis to be newberyite (magnesium hydrogen phosphate trihydrate; $\mathrm{MgHPO}_{4} \cdot 3 \mathrm{H}_{2} \mathrm{O}$ ), an essential constituent in animal urinary calculi that precipitates at $\mathrm{pH}$ below 6 and is commonly found as spherules (Fig. 3b) (Suryawanshi \& Chaudhari, 2014). Similarly, crystals of strontium oxalate, an isomorphic compound of weddelite, grew in an agar gel containing strontium chloride and covered with a solution of oxalic acid, both at $2 M$ (Fig. 3c) (Dalal \& Saraf, 2011). Oxalate crystals formed at similar growth rates for all $\mathrm{pH}$ values tested, with regular bipyramidal and irregular crystalline shapes, regardless of acidic or basic conditions (Fig. 3c).

To test for the effects of urea concentration on the formation of urinary stones and possible treatment to dissolve them, urea was crystallized by evaporation in saturated and supersaturated solutions at room temperature (Fig. 1c). While urea crystals are not directly responsible for urolithiasis, increased urea concentration and its subsequent decomposition in the presence of certain bacteria is an underlying cause of bladder stone formation (Miano et al., 2007). As an indication of the critical concentration of sodium bicarbonate $\left(\mathrm{NaHCO}_{3}\right)$ that would cause crystal dissolution, urea crystals dissolved rapidly in $0.25-1.0 \mathrm{M}$ solutions of $\mathrm{NaHCO}_{3}$, suggesting a possible treatment of bicarbonate tablets to dissolve urinary stones. However, at lower $\mathrm{NaHCO}_{3}$ concentrations (0.02 and $0.125 \mathrm{M}$ ), urea crystals dissolved initially but reappeared after four days as a large single crystal (Fig. 1d). The reason for the reappearing crystal is unknown at this point, but PXRD analysis did not show traces of $\mathrm{NaHCO}_{3}$ in the crystal structure (data not shown).
The second example project, entitled Macro Crystal Growth in Superhydrophobic Systems, was carried out by Daniel Carelli, Liam Kirwan and Keerthana Prakash, freshman students majoring in biology and engineering at NYU Abu Dhabi. The project characterized the effects of a superhydrophobic system on the nucleation of crystals. A superhydrophobic surface is one in which the contact angle of water on the surface is above $150^{\circ}$ because of a low surface energy and a nano-/micro-rough surface (Wang \& Jiang, 2007). This forces water to bead-up and roll off the surface. An example is the lotus leaf, where the waxy nanostructure on the leaf's surface forces water off and carries with it dirt and dust that impedes its ability to carry out photosynthesis (Barthlott \& Neinhuis, 1997). To investigate crystal growth on superhydrophobic surfaces, copper(II) sulfate pentahydrate $\left(\mathrm{CuSO}_{4} \cdot 5 \mathrm{H}_{2} \mathrm{O}\right)$ was chosen, because its bright-blue color makes it easy to detect visually (Fig. 4a). Plastic and glass containers were coated twice with Rustoleum's NeverWet paint to increase the surface tension. After the paint cured, a saturated solution of $\mathrm{CuSO}_{4}$ was used to grow macrocrystals by evaporation (Holden \& Morrison, 1982; Holden \& Singer, 1960). In a glass test tube with a large vertical-surface-area-tovolume ratio, $\mathrm{CuSO}_{4}$ crystallized along the surface of the test tube, but not when the surface is coated with superhydrophobic paint (Figs. $4 a$ and $4 b$ ). The superhydrophobic treatment appeared to inhibit crystal growth on the surface of the container; nevertheless, crystals accumulated at the bottom of the test tube, although not at its sides (Fig. $4 b$ ). These results were also observable in the uncoated test tube, albeit to a lesser extent. Along with the ability of the paint to inhibit surface nucleation, an increase in the rate of crystal growth was qualitatively observed in the superhydrophobic system. In the same time period, more water was qualitatively observed to evaporate from the superhydrophobic system in comparison to the control. This is due to the higher energy associated with contact between water molecules and the superhydrophobic walls.

To gain a better understanding of the extent of hydrophobicity of the commercial coating, the coated glassware was analyzed using scanning electron microscopy (SEM) as well as
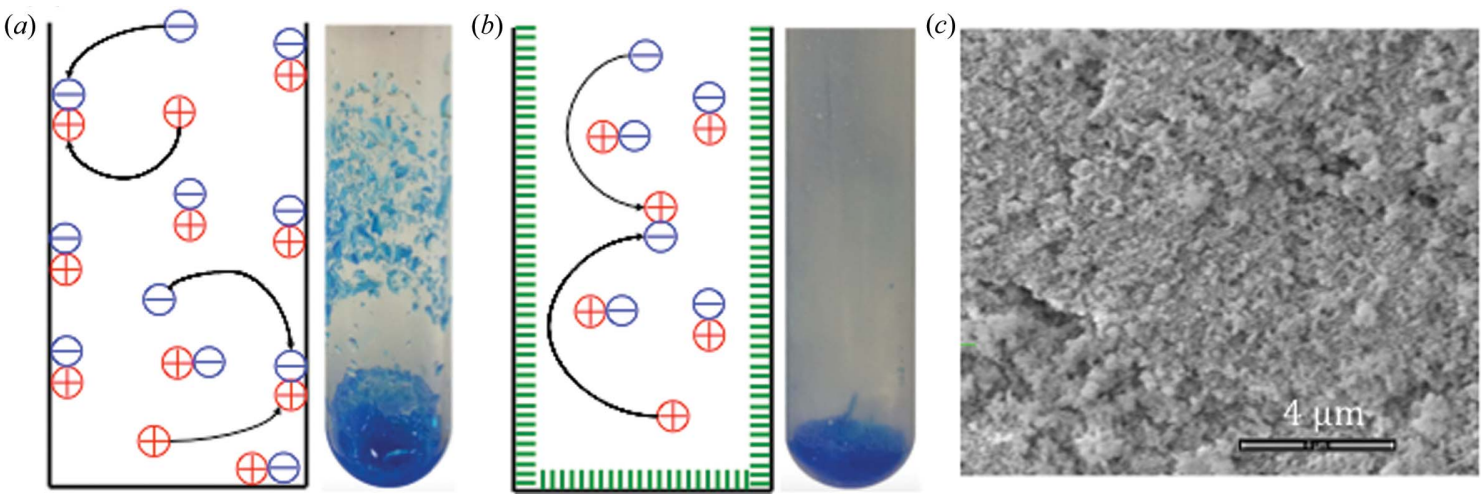

Figure 4

Crystallization on superhydrophobic surfaces. (a) Graphical view of crystallization in a container, showing nucleation sites both in solution and along the sides of an uncoated glass test tube for $\mathrm{CuSO}_{4}$ crystals. (b) Crystal growth in a container coated with superhydrophobic paint, indicating the surfaces' nano-roughness. Nucleation was observed only in solution but not along the container walls. (c) SEM image of the superhydrophobic coat on a glass test tube, showing the nano-rough surface structure. 
contact angle measurements (Fig. 4c). The SEM revealed nanostructures with sizes less than $100 \mathrm{~nm}$, consistent with what is required for superhydrophobicity according to our literature research (Fig. 4c) (Lin et al., 2013). Additionally, we measured the contact angle to confirm the degree of hydrophobicity. These experiments helped the students understand how these characteristics are determined and better informed us on the material we were studying (for further details, the students' poster is included in the supporting information).

\section{Expanding horizons}

This laboratory is designed to introduce students to the world of crystals and crystal growth. Having obtained crystals, there are a fascinating variety of experiments for characterization and further study that may be carried out, depending on the time and facilities available. Just a few of these are noted here.

\subsection{Microscopic examination}

A stereomicroscope with digital photographic capability provides the opportunity for detailed examination of the crystals obtained and the documentation of all the results for laboratory reports, poster presentations and seminars. A highly recommended upgrade for the stereomicroscope is a rotating polarizing stage and analyzer. This can be used to distinguish between amorphous and crystalline materials, and to determine the birefringence and index of refraction of the crystals. Another step up is a hot stage attachment to study the thermal behavior of the solids obtained. Precise determination of melting point is possible, and phenomena such as phase changes, desolvation and dehydration can be readily observed and recorded with such a device, often providing dramatic photographs and videos. For demonstration and observation by more than one or two people, a video recorder with an appropriate monitor may be attached.

The crystal structures of all materials used here can be found in the literature. The crystallographic information files (CIF or mmCIF) are available from archival databases such as the Cambridge Structural Database (Groom et al., 2016; https://www.ccdc.cam.ac.uk), the Inorganic Crystal Structure Database (https://icsd.fiz-karlsruhe.de) and the Protein Data Bank (Berman et al., 2000; https://www.rcsb.org) - all with appropriate plotting programs for rendering the structures in a variety of ways - or the laboratory can create a library of the structures studied for ready student access. Many of these programs can also create idealized crystal habits for comparison with those obtained in the laboratory. Features such as the coordination number and geometry of metal cations are readily recognized and presented.

Desktop PXRD instruments are becoming available in many chemistry departments. If such an instrument is available, students can obtain the powder diffraction pattern and compare it with that calculated from the known crystal structure extracted from the databases noted in the previous paragraph. Alternatively, one can create a library of those diffraction patterns and provide them to the students for comparison with the pattern calculated from the CIF.

\section{Challenges for instructors}

In traditional chemistry laboratory sessions, all students typically perform the same experiment, especially for larger classes. Operationally, this makes sense as it is far easier to prepare for, with every bench receiving the same amount and type of glassware and equipment and the required chemicals prepared in bulk; any pre-laboratory discussion is on the same topic for all. For the students, it is easier as they have each other to reach out to in times of uncertainty, or worse, lack of preparedness. On the other hand, the crystal project which does not provide step-by-step instruction to the students gives them more freedom in the design and overall theme of their projects, where they can personalize the experiments to fit their educational background and specific interests. This removes all traditional safety nets for instructors in terms of operational ease and poses a unique set of challenges, the first and most obvious of which is the unique preparation for each laboratory group as different groups run different projects and require different experimental setups; the second is the need to prepare an appropriate pre-laboratory lecture, and, last, is the need to help prepare students who are struggling with their project. Having said that, the crystal project was a great success at the different NYU campuses, where the majority of the instructors are chemistry or biology graduates with no prior X-ray crystallography experience (Supplementary Note 3). Our experience demonstrates that it is feasible for this laboratory exercise to be conducted by graduate teaching assistants, which has been reflected by the response of the different instructors of the course (Supplementary Note 4). While there are certainly challenges in planning and running the crystal project, the greatest benefit to the instructors involved over the years is observing the self-motivation that the students seem to find when carrying out their own experiments.

To overcome these challenges, preparation has been found to be critical. Students are afforded a list of available and suitable (i.e. safe) chemicals (Table S1) from which they prepare an outline of their project, a critical component for student planning and for the instructor to comment on the project feasibility (in terms of chemicals and equipment). Since it is practically impossible for an instructor to know everything about all of the projects, students should be guided in the art of literature research and independent critical thinking, ideally in the early stages of the laboratory course, in the run-up to the crystal project. In our experience, the best an instructor can do to help students is to ask the right questions, to both ensure students understand what it is they actually want to do and help them determine how best to proceed. Following the instructor's feedback on the outline, a proposal then serves to narrow down the possible options and give the instructor a better handle on the theoretical and practical concepts behind the projects; it is quite common to notice that there are several projects which plan on testing similar factors 
(e.g. $\mathrm{pH}$, temperature and solvent effects, to name a few). It has been found helpful to re-emphasize the independent nature of the assignment at each stage of feedback to students. We have also found the role of the instructor evolving from being a 'sage on the stage' to more of a 'guide by your side', and reminding students of this puts an emphasis on learning through discovery as opposed to being dictated to by the instructor.

\section{Evaluation and assignments}

Students are evaluated at different stages of the project, including the crystal outline and proposal that are due before the start of the project (Table S2). The instructor comments on the crystal outline will help the students to draft a full proposal that is another evaluation tool to determine the students' ability to search literature, draft their ideas into a project and design experimental protocols. The proposal is graded in terms of novelty of ideas, ability to draft a full project with expected outcomes, incorporation of literature, proposal format and project timeline. Another important evaluation tool is the students' performance and adherence to laboratory etiquettes, which include good record keeping and data management. The laboratory notebook is also a valuable tool to determine the students' ability to organize and manage their data and to evaluate their performance in the laboratory. Finally, a full report similar in format to a scientific research article is due prior to the Crystal Growth Symposium, an end of semester celebration for the students to showcase their work and for different faculty members and instructors from chemistry and other disciplines to evaluate the students' projects. Scores from the judges are incorporated into the students' final grades and used to select the top three posters for prizes and recognition.

\section{Supporting information}

The supporting information contains the pre-laboratory lectures, an outline of the laboratory schedule and assignments, a list of recommended chemicals to be used in the crystal project, and samples of students' posters submitted in the fourth and fifth crystal symposium at NYUAD. This material is available at https://doi.org/10.1107/S1600576718009573.

\section{Acknowledgements}

The authors especially express their appreciation to the assistant instructors involved in the teaching of the laboratory course, including Diana Rishmawi and Elizabeth Latham. The authors are also grateful to the Foundation of Science 3 and 4 laboratory sections for data and feedback collected in spring semesters from 2011 to 2015 . Special thanks to the following students for contributing their crystal pictures and posters: Liam Kirwan, Keerthana Prakash, Christos Zoukos, JooHee
Choi, Hassan Ahmed Mahmoud, Joel Ntwali Rukazambuga, Sharon Qiu, Kyler Meehan, Farzan Ahmad Khan, Yaereen Dho, Prasant Adhikari, Zoha Alvi, James Carrington Gardner, Hassan Nahas, Yumi Gambrill, Anna-Lisa Hennig, Samuel Ridgeway, Rasha Shraim, Ieva Liepuoniute and TingChe Lin. We would like to thank James Weston and Liang Li for giving demonstrations and running students' samples using the following equipment and techniques: optical microscope, energy-dispersive X-ray spectroscopy, contact angle measurement and scanning electron microscope. Part of the students' project was carried out using Core Technology Platform resources at NYU Abu Dhabi.

\section{References}

Barthlott, W. \& Neinhuis, C. (1997). Planta, 202, 1-8.

Berman, H. M., Westbrook, J., Feng, Z., Gilliland, G., Bhat, T. N., Weissig, H., Shindyalov, I. N. \& Bourne, P. E. (2000). Nucleic Acids Res. 28, 235-242.

Byrappa, K. \& Ohachi, T. (2003). Crystal Growth Technology. Berlin: Springer.

Dalal, P. \& Saraf, K. (2011). Bull. Mater. Sci. 34, 377-382.

Dhanaraj, G., Byrappa, K., Prasad, V. \& Dudley, M. (2010). Springer Handbook of Crystal Growth. Berlin: Springer.

Domin, D. S. (1999a). J. Chem. Educ. 76, 109-112.

Domin, D. S. (1999b). J. Chem. Educ. 76, 543-547.

Douglas, B. \& Ho, S. M. (2007). Structure and Chemistry of Crystalline Solids. New York: Springer.

Grazulis, S. et al. (2015). J. Appl. Cryst. 48, 1964-1975.

Groom, C. R., Bruno, I. J., Lightfoot, M. P. \& Ward, S. C. (2016). Acta Cryst. B72, 171-179.

Henisch, H. K. (1996). Crystal Growth in Gels. New York: Dover Publications.

Hodson, D. (1990). Sch. Sci. Rev. 70, 33-40.

Holden, A. \& Morrison, P. (1982). Crystals and Crystal Growing. Cambridge: The MIT Press.

Holden, A. \& Singer, P. (1960). Crystals and Crystal Growing. New York: Anchor/Doubleday Publishing.

IUCr (2018). 2018 IUCr Crystal Growing Competition for Schoolchildren, https://www.iucr.org/outreach/crystal-growing-competition2018.

Khan, S. R. (1997). World J. Urol. 15, 236-243.

Lin, J., Chen, H., Fei, T. \& Zhang, J. (2013). Colloids Surf. A Physicochem. Eng. Asp. 421, 51-62.

McPherson, A. (2004). Methods, 34, 254-265.

Miano, R., Germani, S. \& Vespasiani, G. (2007). Urol. Int. 79 (Suppl. 1), 32-36.

Rao, N. P., Preminger, G. M. \& Kavanagh, J. P. (2011). Urinary Tract Stone Disease. London: Springer.

Reid, N. \& Shah, I. (2007). Chem. Educ. Res. Pract. 8, 172-185.

Smith, C. L. \& St Peter, J. V. (2007). Urol. Res. 35, 243-246.

Sun, W. D., Wang, J. Y., Zhang, K. C. \& Wang, X. L. (2010). Res. Vet. Sci. 88, 461-466.

Sunagawa, I. (2005). Crystals: Growth, Morphology, and Perfection. Cambridge University Press.

Sunagawa, I. \& Suchtelen, J. V. (1995). Morphology of Crystals. The Netherlands: Springer.

Suryawanshi, V. B. \& Chaudhari, R. T. (2014). J. Cryst. Proc. Tech. 4, 212-224.

Syme, H. M. (2012). Arab. J. Urol. 10, 230-239.

Wang, S. \& Jiang, L. (2007). Adv. Mater. 19, 3423-3424. 